\title{
Labyrinthe
}

$36 \mid 2011$ (1)

«Par les Grecs »

\section{Warum Greece ?}

\section{Pierre Judet de la Combe}

\section{OpenEdition}

Journals

Édition électronique

URL : http://journals.openedition.org/labyrinthe/4128

DOI : $10.4000 /$ labyrinthe. 4128

ISSN : 1950-6031

Éditeur

Hermann

Édition imprimée

Date de publication : 15 février 2011

Pagination : 81-88

ISBN : 9782705680541

\section{Référence électronique}

Pierre Judet de la Combe, « Warum Greece? », Labyrinthe [En ligne], 36 | 2011 (1), mis en ligne le 01

février 2013, consulté le 30 avril 2019. URL : http://journals.openedition.org/labyrinthe/4128; DOI

10.4000/labyrinthe.4128

Propriété intellectuelle 


\section{Warum Greece?}

Pierre JUDET DE LA COMBE

Certainement pas parce que ce serait nous-mêmes en plus lointains, en antiques et d'origine; ni, car c'est aussi convenu, parce ce serait quelque chose d'Autre, de perdu et d'un peu barbare, loin des illusions dont il faudrait sans cesse se déprendre pour mieux le connaître; ni, encore, l'obligation ardente de s'y transporter comme à l'étranger pour en revenir comme vraiment soi-même, c'est-à-dire intempestif car un peu antiquisé et par là enfin actuel et renouvelé - selon les trois formes complémentaires d'extase qui ont cours dès qu'on parle de Grèce (antique). Tout simplement parce qu'il est dur de donner un sens à ce mot. Surtout si on n'est pas historien et qu'on ne parle pas en termes d'époque, mais qu'on est philologue et qu'on lit des textes, un par un, sans présupposer qu'ils entrent nécessairement dans un tout plus ou moins harmonieux. La « Grèce » devient une simplification inventée, un abus de langage ou une chose déjà dite qu'il faudrait seulement creuser à l'infini et retrouver dans chacune de ses œuvres, sans grande surprise. La Grèce, la mentalité grecque, l'homme grec sonnent alors faux, même si, à la réflexion, il y a là, sous certaines conditions, des constructions scientifiques possibles, puisque l'Ancien doit bien exister d'une manière ou d'une autre. Mais pourquoi chercher, d'abord, à généraliser, à retrouver du Même, de la cohérence unifiante, même quand on dit que la Grèce nous est autre? Même ainsi, elle fait bloc, et elle devient un objet parmi d'autres, pour la seule connaissance ou, si on y tient, pour l'admiration, qui n'est jamais absente, sauf pour les femmes et les esclaves, chez ceux qui professent son altérité d'ensemble et refusent l'idée d'un « miracle grec ».

Au départ, quand on lit des poètes grecs, c'est sans doute plus simple. Homère, Pindare, Euripide, Aristophane ou Théocrite attirent parce que c'est grandiose et qu'on ne sait d'abord pas pourquoi. Ce sont des « autres », pas seulement par rapport à nous, mais en soi, par rapport aux autres textes de leur temps, par rapport à leur réception et par rapport à eux-mêmes, dans leur variance et opacité incessantes. Avec cette difficulté ou intérêt de plus que cette altérité intrinsèque, individuelle, des œuvres ne peut pas être abordée directement. Car ce n'est pas « la Grèce », ou 
un bout d'elle, et encore moins un objet sur lequel on tomberait, mais, déjà, de l'hypersélectionné, du canonique dès l'Antiquité, choisi par les philologues d'alors à Alexandrie. Ils sont, avec les autres poètes du canon, tout ce qui nous reste, ou presque, sans comparaison possible. C'est de l'admiration forcée, sédimentée et indéfiniment répétée; une tradition. Il ne s'agit alors pas de la prolonger ou de la récuser par souci moderne d'objectivité, mais de l'interroger en se demandant de quoi ces textes sont faits et, surtout, ce qu'ils font vraiment face à cette canonisation qui les a sauvés en même temps qu'elle les a figés. Avec l'idée, puisqu'on les lit encore, qu'il y a bien là de quoi, qu'ils valent la peine et que la sélection, aussi idéologique qu'elle ait été, réductrice et éloignée de la réalité historique d'origine de ces textes, était aussi une forme de reconnaissance, qu'elle avait, à sa manière, répondu à une prétention de ces œuvres, répondu à quelque chose de normatif, à une manière de poser ce que peut être la poésie. Non pas la réussite ou l'excellence, qui supposent des normes immuables, mais une façon singulière de proposer des normes, d'y prétendre.

Lire devient l'expérience de la confrontation entre les jugements, nécessairement généraux, qui ont construit cette admiration historique et la précision du dire qui a fait ces œuvres, ses tensions internes, sa problématisation constante comme support de la lettre. C'est avancer avec des concepts, nécessairement hérités d'abord, sur la langue, le genre, le rythme, les idées, et avec la révision de ces concepts qu'impose la résistance de la matière. Il n'y a là rien de grec, puisque la même expérience vaut pour les canonisés plus récents, Boccace, Henry James, Henri Michaux ou Claude Simon. C'est tout bêtement lire. Ce qui reste de proprement grec est l'effet des œuvres anciennes sur les traditions théoriques et scientifiques qui depuis l'Antiquité encadrent la lecture dans un débat contradictoire ininterrompu sur ce qu'est le sens d'un texte et qui, pour nous, se sont condensées dans les corpus théoriques véhiculés par les langues européennes; pour simplifier trop: l'anglais des empiristes et l'allemand des romantiques et de leurs héritiers, avec depuis la seconde moitié du $\mathrm{Xx}^{\mathrm{e}}$ siècle, la langue des sciences sociales à la française.

La Grèce historique n'est alors plus un objet, mais seulement une condition qui a fait que la langue, les idées, les croyances, les pratiques de la communication, etc., étaient, à l'époque, telles et non pas autres, qu'elles étaient donc un donné, le medium où les œuvres pouvaient tenter de faire sens. Faire œuvre consistait non pas à exemplifier seulement ce 
milieu commun, mais à proposer, dans des situations précises et fortement codifiées, une synthèse particulière (puisque «être le meilleur» était chaque fois visé) de ces différents matériaux. Et cela ne suppose pas qu'il y ait eu une "Grèce », une synthèse de ces synthèses singulières que sont les œuvres. Le penser, c'est faire l'hypothèse d'une unité préétablie, d'un consensus, la société ne cessant de se mirer elle-même dans ses productions, selon une conception pauvre et aplatie de la réflexion, sans distance, comme jeu purement immanent et donc sans histoire. $\mathrm{Si}$ une telle idée en fait romantique de la Grèce comme totalité, comme cohérence obligée, idée revue et renforcée parfois par certains usages des sciences sociales comme sciences des systèmes, devait servir de modèle ou simplement d'objet pour l'enseignement, on ne serait pas loin d'un éloge réactionnaire du conformisme et du ressentiment contre la liberté possible des textes.

D'où, plutôt, pour le lecteur, l'insistance sur les différences, sur le caractère non nécessairement réconcilié avec soi-même d'une culture ou même d'une œuvre, et le pari que si la langue est de part en part historique, située, et, pour l'époque archaïque, fortement formatée, le rapport à la langue que suppose l'activité de composition porte en soi une dimension transhistorique qui rend la lecture possible. Non pas le génie individuel, mais le simple fait que quelque chose soit dit, dans une forme tenue, soit assumé et construit. Une proposition articulée. Si une œuvre pouvait faire événement, et être transcrite, transmise et imitée, ce n'était pas seulement qu'elle était conforme aux attentes de son public (attentes que nous ne connaissons pas, et que nous ne retrouvons que dans les œuvres, dans la manière dont elles y répondent et, souvent, les thématisent), mais parce qu'elle était nouveauté, et permettait de se représenter autrement tout ce qui avait déjà été dit. Le public devait aussi aimer ça. L'objet, pour nous, devient ce rapport singulier à la tradition dans sa réalisation même, ce mouvement qui lui est donné, qui la fait vivre et qui fait l'histoire. Avec donc ce paradoxe: ce qui fait la nature historique de ces œuvres, comme événement venant ajouter ou modifier quelque chose dans une situation donnée, avec ses codes, est précisément cette dimension transhistorique, c'est-à-dire non réductible à cette situation. C'est cette dimension d'un travail accompli par les œuvres sur les matériaux historiques que sont les différents codes anciens qui ouvre l'histoire, crée du nouveau et donne la possibilité, au moins partielle, de s'y rapporter après coup. 
Mais dire tout cela n'est encore que précautionneux, méthodique, et ne dit rien de l'intérêt d'un chant d'Homère ou d'une ode de Pindare ou de son rival Bacchylide, qui sont quand même des Grecs. S'ajoute alors l'idée qu'il s'agit bien d'art et de poésie, malgré certaines dénégations scientifiques récentes qui se refusent à utiliser pour les œuvres archaïques des catégories modernes comme « littérature » ou " poésie » et qui demandent de s'en tenir aux seules catégories indigènes et donc de ne pas oublier que ces œuvres « poétiques » faisaient d'abord partie de cérémonies religieuses et politiques. Certes. Mais les Grecs archaïques disaient « la Muse », et, par là, parlaient bien de poésie. Nos distinctions actuelles culte $v s$. poésie, arts premiers $v s$. arts réfléchis et dotés d'une esthétique consciente, ne tiennent pas la route dès qu'on s'intéresse aux matériaux travaillés par les différentes activités démiurgiques, que ce soit dans le contexte d'un culte vivant, d'une activité lettrée de cabinet ou au théâtre. Le langage, et non seulement la finalité sociale d'une activité (ou plutôt l'idée abstraite qu'on s'en fait, puisque le langage est bien social et fonctionnel) imposait ses contraintes et ses potentialités. Et John Dewey nous a rappelé que l'esthétique n'est pas un domaine séparé, mais un mode d'expérience dans le travail sur le matériau et donc qu'un objet de culte pouvait aussi être de l'art. Si on accepte cela, une histoire longue, et pas seulement grecque mais comparative, s'ouvre alors. Elle montre comment des grammaires langagières universelles ou universalisables se réalisent dans des formes historiques particulières et contrastées : comment la prédominance du « il» dans le récit épique («Patrocle tua Sarpédon ») et la distanciation qu'il suscite, au point que l'histoire racontée se réfère, en Grèce, à une époque mythique définitivement passée et close, fermée au présent, permettent, dans la tension constante avec le « maintenant» et le « je » ou le « nous » de la performance poétique comme éléments de l'histoire présente, exclue du mythe, une réflexion sur la tradition narrative, sur le mythe, les dieux, sur leur puissance et leur réalité; l'épopée déréalise en même temps qu'elle pose un monde objectif et total; comment le « je » conventionnel de la poésie lyrique de circonstance (passage à la puberté, mariage, victoire athlétique, mort) peut se construire, différemment selon les genres et les poèmes, comme événement langagier individuel où se recompose le sens des éléments mythiques, politiques ou éthiques mobilisés pour le moment de la performance, qui devient, selon les poétiques des poèmes, ou bien temps de rupture ou bien étape d'une continuité historique possible définie par 
l'œuvre; le « je » posé dans le poème est un programme, diversement réalisé; ou comment la situation basique qui définit universellement le théâtre comme coupure entre des acteurs qui jouent et un public qui ne joue pas peut devenir le moteur d'une réflexion dramaturgique au sein des œuvres, réflexion de métier qui transforme les contenus mis en scène et différencie les manières de faire des auteurs. Seule alors la comparaison des œuvres, des genres, des époques, grecques et autres, offre des repères.

Mais, malgré tout, n'y a-t-il pas dans tout cela quelque chose de spécifiquement grec? Les Grecs n'ont-ils pas, avec au moins les Latins, fait époque? Ou faut-il renoncer à la distinction habituelle entre l'Ancien, le Moderne et le Contemporain? Certains hellénistes, surtout dans les pays anglo-américains, disent que oui. Que les œuvres poétiques et théoriques de l'Antiquité valent ou ne valent pas, que c'est à voir cas par cas, sans se laisser impressionner par l'idée d'une histoire progressive et nécessaire qui ferait de «l'homme grec », poète ou philosophe, un être différent de nous, ou bien plus arriéré et moins bien équipé intellectuellement, ou simplement autre. Ce qui compte est ce qu'il dit, qu'on doit prendre au sérieux, et on se dit à même de le comprendre directement si on est méthodique, à même de le corriger, de le restituer à la lettre ou même de l'améliorer si pour une raison ou une autre les textes transmis sont défaillants (c'est ainsi que souvent on édite maintenant les poètes grecs, en les retouchant). Quant aux concepts théoriques qu'on trouve dans ces textes, ils seraient, à l'examen, souvent plus valides que bien des concepts modernes, surtout en matière d'éthique et de politique. Il n'y a pas de rupture nécessaire entre les époques, pas de progrès ou d'altérité. D'où le retour à Aristote. Une première objection, facile, contre cette négation de l'histoire est que si Aristote s'avère finalement plus performant que Kant en morale, il reste qu'il n'a jamais eu à envisager et à critiquer une position comme celle de Kant, et donc qu'il y a bien, au moins, deux époques théoriques différentes. Plus fondamentalement, c'est traiter les œuvres théoriques comme une série d'énoncés plus ou moins rationnels, sans vraiment se demander comment ces énoncés sont possibles dans un contexte d'ensemble, dans le tout que forme une pensée. On est donc bien renvoyé à la question du pourquoi telle ou telle position théorique n'a pas eu cours alors ou pourquoi elle a été minoritaire, contestataire, alors qu'elle régnait ailleurs. Il y a bien des «types idéaux » de pensée, transculturels et transépoques, mais ils s'organisent, se légitiment différemment selon les époques et les cultures. 
Est-on alors seulement renvoyé à l'idée d'une consistance de la Grèce ancienne non pas comme Autre mais, de manière déterminée et historique, comme ce qu'ont nié les Modernes et les Contemporains, comme ce dont ils se sont détachés non par ignorance ou différence intrinsèque, mais par discussion et réflexion? L'Antiquité, à l'opposé de nous, poserait selon cette perspective un monde organisé par les dieux mais sans transcendance et sans salut (à part dans des pensées marginales comme celle des cultes de Dionysos); un monde éthique et politique où la liberté n'est pas un principe absolu, fondateur, qu'il soit posé ou critiqué, mais seulement une condition objective, ne pas être esclave ou soumis à un tyran; un monde qui se pense comme réalité fermée, comme cosmos, contraire au monde ouvert des Modernes comme univers infini (à part dans des pensées critiques comme celle des Atomistes)?

Il y a bien cela, qui a déjà été dit mille fois, mais non comme structure de sens imposée, comme donné, mais comme l'élément d'un problème général qui traverse en permanence les différentes périodes de la culture grecque antique : celui d'une tension toujours renouvelée entre, d'une part, des constructions sémantiques closes, en correspondance avec ce monde pensé comme achevé, limité, et, de l'autre, le moyen de les élaborer, à savoir le langage, qui est par nature, dans le jeu infini entre ses diverses fonctions, toujours plus ouvert que les constructions qu'il produit. L'intérêt se porte alors non pas seulement vers les résultats positifs de cette activité, sur les œuvres où elle se condense, que ce soient les normes juridiques, les constitutions politiques, les systèmes théoriques et leurs principes ou les poèmes comme organisations sémantiques achevées, mais aussi sur les conditions langagières de ces constructions historiques. Ce n'est pas l'un ou l'autre, ou bien l'attention pour la cohérence des œuvres historiques, pour le caractère déterminé de leur sens, ou bien le rappel de la puissance du langage comme instance ouverte d'indétermination, comme déconstruisant, puisque cette dichotomie suppose une idée trop courte, trop peu différenciée du langage. Ce qui est à l'œuvre est bien plutôt cette tension avec ses deux pôles, tension qui produit les textes et leur détermination, et en fait des événements potentiellement nouveaux.

Avec cet avantage énorme pour la culture grecque dans son devenir (la « Grèce », donc), que l'on peut suivre pas à pas, depuis Homère et Hésiode, l'histoire des modes de systématisation, de construction des univers de sens, depuis le mythe comme récit systématique jusqu'à la théorie de la nature, la théorie du politique et de l'histoire. Avec l'obligation, puisque 
pendant longtemps le mythe n'a eu d'interlocuteur que lui-même, avant que la philosophie n'intervienne, de comprendre des formes de totalisation sans fondement conceptuel mais liées aux procédures de la composition poétique. Ce n'est donc pas une émergence de la raison qu'il s'agit d'identifier, comme si le mythe ne pouvait pas être rationnel, mais des formes différenciées de traitements du langage comme porteurs de rationalité. Cela nous renvoie aux questions d'une théorie générale des modes de savoir comme produits historiques, en Grèce et ailleurs dans le monde.

Et cela d'autant plus que cette époque est close et prend par là tout son sens actuel. Rien d'elle ne pourra se répéter, sauf sur un mode ironique et décalé, et cette tension ouverte dont nous parlons a fait son temps, sous ces formes-là. Elle nous apparaît à travers une culture qui s'est achevée, et qui prend par là une signification universelle comme moment singulier et déterminé d'un travail historique proliférant. Il n'y a là rien d'exemplaire, aucune valeur en soi, mais l'occasion, en raison de la coupure qui nous sépare de ce passé qui est devenu une singularité à part, de cerner mieux notre moment.

Celui-ci est désormais infiniment démultiplié et mondialisé, même dans son rapport à « notre » tradition antique. L'Antiquité grecque, ou latine, n'est, en effet, qu'une parmi beaucoup d'autres, puisque toutes les civilisations se sont donné leur Antiquité et vivent dans une sorte de co-linguisme qui les structure, dans une relation perpétuellement refaite et changeante entre la langue de tous les jours et les langues savantes du passé, plus ou moins mortes. Pour « nous », ces langues sont essentiellement le latin et le grec, avec l'hébreu et l'arabe classique, mais ce sont aussi les états anciens et savants de notre langue ou des différentes langues du territoire (dans les limites qu'on voudra, selon les histoires de chacun), tels qu'ils se sont déposés dans les littératures et dans les sciences. Mais même dans cette relation à ce passé limité, réel ou reconstruit, dont chacun peut se réclamer, nous avons quitté le face à face avec notre seule tradition, puisque sommes pris dans un contexte de traduction et retraduction permanentes entre les langues et les cultures d'un espace désormais universel. Or ce contexte d'échange ne concerne pas seulement les langues dans leur état d'aujourd'hui, qu'il s'agirait de connaître, mais les langues comme puissances de production, comme histoire, comme étant portées et renouvelées par la relation que les sociétés établissent avec les Antiquités plus ou moins anciennes qu'elles se sont choisies. La Grèce, comme histoire singulière, n'est que l'une de ces références 


\section{Labyrinthe, $n^{\circ} 36$}

anciennes, comparables aux autres non par ses contenus seulement, mais par les modes de liens, de réappropriation que les époques plus récentes jusqu'à aujourd'hui ont établis avec elle. S'y intéresser prend sens dans un échange global sur les types de rapport au passé comme condition d'une entente dans le présent. Les « Humanités » sont elles-mêmes globales. 